\title{
Nanomechanical structure-property relations of dynamically loaded reactive powder concrete
}

\author{
P. G. Allison ${ }^{1}$, R. D. Moser ${ }^{2}$, M. Q. Chandler ${ }^{1}$, T. S. Rushing ${ }^{1}$, \\ B. A. Williams ${ }^{1} \&$ T. K. Cummins ${ }^{1}$ \\ ${ }^{1}$ US Army Engineer Research \& Development Center, USA \\ ${ }^{2}$ Georgia Institute of Technology, USA
}

\begin{abstract}
Low water-to-cement ratio $\left({ }^{\mathrm{w}} / \mathrm{c}\right)$ reactive powder concretes (RPCs) exhibit much higher compressive strengths compared to conventional concrete through optimized particle packing and specialized curing regimes. The high strain-rate impact behavior of RPCs was investigated at the macroscale. However, little work has been done to study the fundamental material behaviors and failure mechanisms of RPC under high strain impact and penetration loads at lower length scales. These high strain-rate loadings have many possible effects on RPCs at the microscale and nanoscale, including alterations in the composition and bonding present in hydrated phases such as calcium silicate hydrate (C-S-H), in addition to fracture and debonding. In this work, the possible chemical and physical changes of RPCs under high strain-rate impact and penetration loads were investigated using a novel technique wherein nanoindentation measurements were spatially correlated with chemical composition using electron microscopy. Results indicate that high strain-rate impacts degrade both the elastic modulus and indentation hardness of RPCs and, in particular C-S-H, with damage likely occurring due to microfracturing and debonding. Additional studies will be required to better understand degradation phenomena within C-S-H itself.
\end{abstract}

Keywords: nanoindentation, SEM, EDX, RPC, UHPC, C-S-H.

\section{Introduction}

Concretes and Portland cement-based materials are the most-produced manmade materials on earth, with over twenty billion tons produced per year. The 
ubiquitous use of cementitious materials within protective infrastructures makes quantifying the response of cementitious materials to high strain-rate penetration and impact loads a critical need as designs become more reliant on computational tools.

Low water-to-cement ratio $\left({ }^{\mathrm{w}} / \mathrm{c}\right)$ reactive powder concretes (RPCs) exhibit superior compressive strength and ductility (when fibers are admixed) compared to conventional concrete and is increasingly being used for high strain-rate loading applications [1]. To fully explore the potential of this material for high strain-rate loading applications, its fundamental failure mechanisms under impact and penetration loads have to be investigated. RPC is a highly heterogeneous material with a microstructure consisting of hydrated cement paste (HCP), unhydrated cement particles, fine aggregates, potentially steel fibers and/or polymer fibers, and pores ranging from nanometer to millimeter in diameter [2]. The addition of pozzolanically reactive silica fume results in an HCP comprised primarily of calcium silicate hydrate (C-S-H).

$\mathrm{C}-\mathrm{S}-\mathrm{H}$ is the most important phase of the Portland cement hydration process and functions as the binding component that holds the various other phases of RPC together. C-S-H consists of physically and chemically bound water in nanometer-scale gels, bulk water in gel and capillary pores, adsorbed water on the surfaces of gels, and may behave nano-granularly [3]. Grady [4] suggests that $\mathrm{C}-\mathrm{S}-\mathrm{H}$ may go through chemical changes such as dehydration or vaporization under shock impact loading. Instrumented indentation; namely nanoindentation, techniques were used to quantify the structure-property relations of concrete at lower length scales. Velez et al. [5] performed nanoindentation tests to quantify the elastic modulus and hardness of synthetically manufactured Portland cement clinker phases. Hughes and Trtik [6] used depth-sensing nanoindentation and energy dispersive X-ray (EDX) analysis to correlate the major phase compositions and mechanical properties of hydrated cement paste.

Ulm et al. [7] developed a novel statistical nanoindentation technique to characterize cement paste and were able to identify the existence of two distinct types of C-S-H, LD C-S-H and HD C-S-H. DeJong and Ulm [8] and Constantinides and Ulm [9] used a similar approach to study the degrading mechanisms of calcium leaching and high temperature on C-S-H. Sorelli et al. [10] also used similar techniques to characterize the properties and volume fraction of different phases in Ultra High Performance Concrete (UHPC). Their research showed that UHPC with a low w/c (0.2) has a much higher volume fraction of HD C-S-H and unhydrated clinker than LD C-S-H compared to concrete with higher w/c.

In this work, the influence of impact loadings on the nanomechanical properties of RPCs is investigated. Specimens were extracted for impacted and non-impacted panels of RPC. A novel technique coupling nanoindentation with spatially correlated scanning electron microscopy (SEM) and chemical analysis using energy dispersive X-ray spectroscopy (EDX) was developed to characterize damage due to high strain-rate impact loadings in the RPC panels. 
Based on the results of these studies, a possible mechanism for microstructural damage in RPCs is proposed, and the caveats associated with the techniques utilized are discussed.

\section{Experimental procedures}

\subsection{Materials}

An RPC mixture with a $\mathrm{w} / \mathrm{c}$ of 0.2 and an unconfined compressive strength of 180 $\mathrm{MPa}$ was cast into panels 305-mm wide by 305-mm long and 25.4-mm thick for ballistic impact testing. The panels were impacted by 11-mm-diameter steel spheres at half the ballistic limit velocity, which was determined according to MIL-STD-662 [11]. The mixing, casting, curing, and ballistic testing is detailed in the manuscript by Rushing et al. [2].

\subsection{Coupled nanoindentation and SEM/EDX analysis}

Analysis of impacted and non-impacted RPC specimens was performed using a novel technique of nanoindentation coupled with SEM imaging and EDX chemical analysis. In this coupled method, a large number of indents are performed over a standardized indentation grid placed on the RPC sample, after which, each indent is spatially correlated using SEM to obtain an image and EDX to determine the chemical composition. The resulting dataset contains the nanomechanical properties and chemical composition along with an image at each indentation site. This coupled method allows for improved differentiation between the various components present in RPCs and can be used to better correlate alterations in nanomechanical (e.g., due to impact loadings) properties to specific microstructural features. Details on specimen preparation techniques and experimental methods are described below.

\subsubsection{Specimen preparation}

Specimens were extracted from the panel in the impacted zone and a nonimpacted zone as shown in Figure 1. The center of the non-impacted location was $38 \mathrm{~mm}$ from both the top edge and side of the panel. This location was selected to minimize the potential for edge effects from casting while also avoiding the damaged zone to the greatest extent. A 25.4-mm-diameter diamondtipped coring bit was used to core the non-impacted specimens, which were then cast into 31.8-mm-diameter cylindrical molds using EpoHeat low-viscosity epoxy supplied by Buehler. The impacted specimen was sectioned using an oilcooled Struers Secotom high precision cut-off saw. The cross section of the specimen was placed into a 31.8-mm-diameter cylindrical mold and mounted in EpoHeat epoxy from Buehler. After the epoxy fully cured, the samples were sectioned in half by the oil-cooled cut-off saw to obtain a cross section from the center of the panel, thus limiting any surface effects such as laitance. 


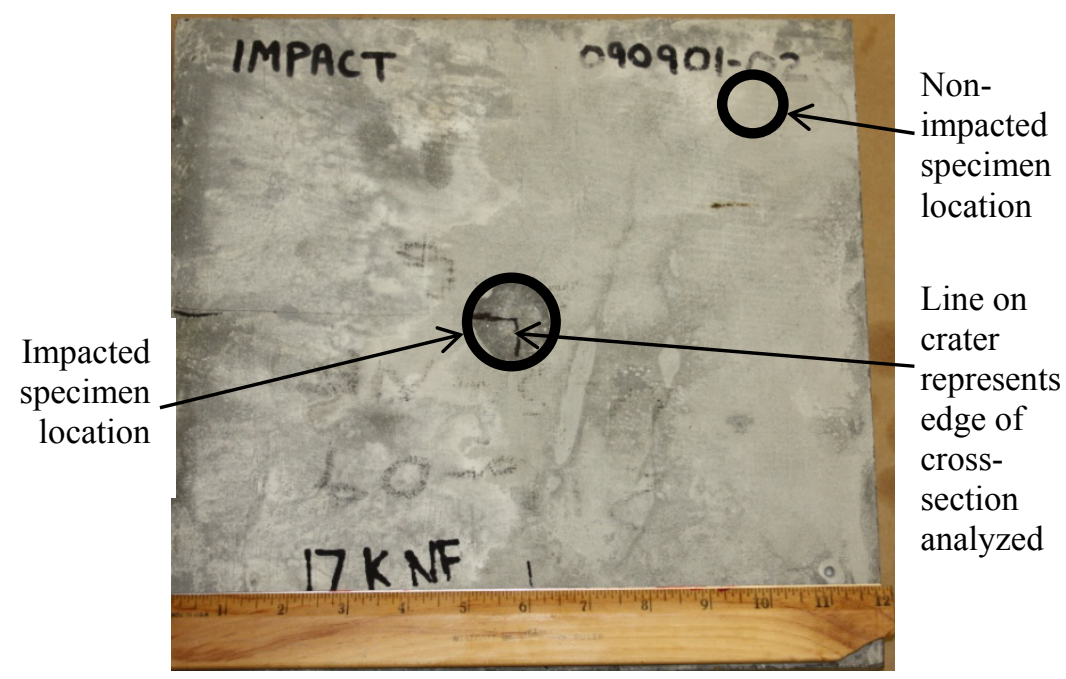

Figure 1: Location of impacted and non-impacted specimens removed by coring from the low $\mathrm{w} / \mathrm{c}$ RPC panel.

A Buehler Ecomet/Automet 250 automatic polishing wheel was used to polish the extracted specimens. The polishing procedure utilized a 240-grit diamond polishing pad at 230 RPM until the specimen was planar, followed by an UltraPad ${ }^{\mathrm{TM}}$ with a 9- $\mu \mathrm{m}$ diamond paste at $130 \mathrm{RPM}$ for 5 minutes, and a third polishing step using a TriDent ${ }^{\mathrm{TM}}$ pad with a $3-\mu \mathrm{m}$ diamond paste for 5 minutes. These first three polishing steps used a 50:50 mixture of ethylene glycol and ethanol for a lubricant. Final polishing utilized a ChemoMet ${ }^{\mathbb{B}}$ pad with $0.05-\mu \mathrm{m}$ alumina in ethylene glycol for 5 minutes. All steps used a force of $30 \mathrm{~N}$ for polishing. Once polished, the samples were desiccated prior to indentation.

\subsubsection{Nanoindentation}

Polished specimens were examined using an Agilent Technologies G200 nanoindenter to probe microstructural changes across the specimens. Indentations were performed using a pyramid-shaped diamond Berkovich indenter with a tip radius of approximately $20 \mathrm{~nm}$. Prior to each measurement, a $2^{\text {nd }}$-order area function calibration was performed using a fused silica reference material. Load controlled indentation measurements were performed up to a maximum load of $2 \mathrm{mN}$ at a loading rate of $0.2 \mathrm{mN} / \mathrm{s}$ followed by a hold time of $5 \mathrm{~s}$ and a $10 \mathrm{~s}$ unloading period. Prior to performing the nanoindentation experiments, each specimen was examined in the SEM to create a "map" of images approximately $3 \mathrm{~mm}$ by $3 \mathrm{~mm}$ near the indentation site. This "map" was then used to find a desired location for the indentation grid (i.e., not containing large voids and/or surface defects).

For each specimen, a total of 500 indents were placed with a spacing of 10 $\mu \mathrm{m}$ in the X-direction and $20 \mu \mathrm{m}$ in the Y-direction. Following the indents performed for nanomechanical measurements, $100-\mathrm{mN}$ fiduciary indents were 
placed at a spacing of $245 \mu \mathrm{m}$ in the X-direction and $20 \mu \mathrm{m}$ in the Y-direction from the first indent to aid in identifying the start, middle, and end of each line of indents so that the indentations could be precisely located using the SEM. Assuming a Poisson's ratio of 0.2 , mechanical properties such as elastic modulus and hardness were determined for the interaction region of the indentation site [12].

\subsubsection{SEM and EDX measurements}

Specimens were examined both before and after nanoindentation using an FEI Nova NanoSEM 630 field emission SEM. This device is equipped with lowvacuum capabilities, making it ideal for examining nonconductive cement-based materials without special sample preparation or metallic coating. Imaging was performed at an accelerating voltage of $10 \mathrm{kV}$ using a backscattered electron (BSE) detector to reveal changes in microstructure and the distribution of phases according to their respective densities. When examined after nanoindentation, the "map" of images was used to generally locate the indentation grid, while the fiduciary indents were used to determine the location of each line of indents such that each indent could be correlated with a location on the image. Point chemical analyses were also performed in conjunction with SEM imaging using a Bruker solid-state EDX detector installed in the FEI SEM. Through proper alignment of the indentation grid facilitated by the fiduciary indents, a standardized point chemical analysis grid was developed that resulted in a dataset of point chemical analyses that were spatially correlated with the location of each indent.

\section{Results and discussion}

The highly variable phase composition and distribution present in RPCs presents a variety of different nanomechanical properties that were sampled during nanoindentation measurements. Figure 2 presents the load vs. depth results of five representative indents corresponding to a fine aggregate, an anhydrous cement grain, C-S-H, and an aberrant test. Here, C-S-H was divided into two phases, namely low-density (LD) and high-density (HD), which was determined through molecular simulations and similar nanoindentation studies [9].

Of particular interest is the faulty or "aberrant" test result shown in Figure 2, which defies the traditional stiffening indentation curve typical for homogenous interaction regions but still exhibits an elastic modulus result similar to a homogenous phase (in this case HD C-S-H). These aberrant tests may present themselves as irregular loading and/or unloading curves (as shown in Figure 2). In heterogeneous cement-based materials, aberrant test results may occur due to the presence of voids, polishing defects, cracking during indentation, and as noted more recently, composite or "nanocomposite" multiphase response of material present in the interaction region of the indent [13-15]. The multiphase response of cement-based materials was a topic of recent discussion in the literature and represents an issue that may diminish the utility of nanoindentation as a quantitative microstructural characterization technique. Thus, it is critical to examine each indentation curve and remove aberrant results if reliable 
quantitative information is desired. All indentation curves in the present study were reviewed, and $8 \%$ in impacted specimens and $24 \%$ in non-impacted specimens were deemed aberrant and removed from the dataset.

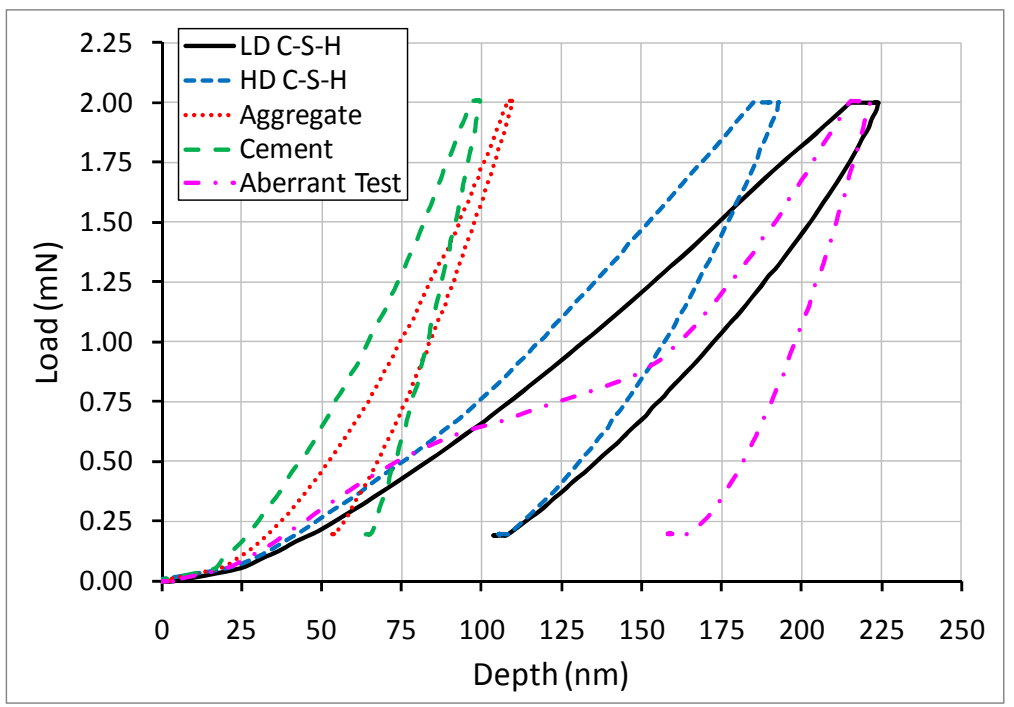

Figure 2: Typical load vs. indentation depth curves for LD and HD C-S-H, aggregate, cement, and an aberrant test result.

Figure 3 (a) illustrates a typical grid of 500 measurement indentation points and fiduciary indents superimposed on a BSE micrograph of an RPC specimen. Using BSE imaging, the anhydrous cement and silica fume can be seen with a bright signature, followed by fine aggregates and silica flour, and finally by the HCP (i.e., solid products of cement hydration with low density) and voids that appear darkest in the image. The HCP appeared to be comprised primarily of C-S-H, with all $\mathrm{Ca}(\mathrm{OH})_{2}$ likely consumed by pozzolanic reactions and subsequently converted into C-S-H. Figures 3 (b) and (c) present contour maps of $\mathrm{Ca}: \mathrm{Si}$ ratio and elastic modulus results, respectively, corresponding to the indentation grid shown in Figure 3 (a). A clear correlation can be observed between the location of the various components of the RPC and their respective composition and mechanical properties.

In addition, the benefits of using this coupled technique are also particularly apparent when trying to differentiate between phases with similar properties. For example, fine aggregate particles and anhydrous cement present in RPCs may exhibit similar mechanical properties, making phase identification/quantification from only nanoindentation results a challenging task. However, when nanoindentation measurements are coupled with chemical composition at the indentation site, the distinction between fine aggregates (with low $\mathrm{Ca}: \mathrm{Si}$ approaching zero) and cement (with high Ca:Si of 5 to 7) becomes clear. Similar comparisons can be made for the various phases of cement hydration present in the HCP. 


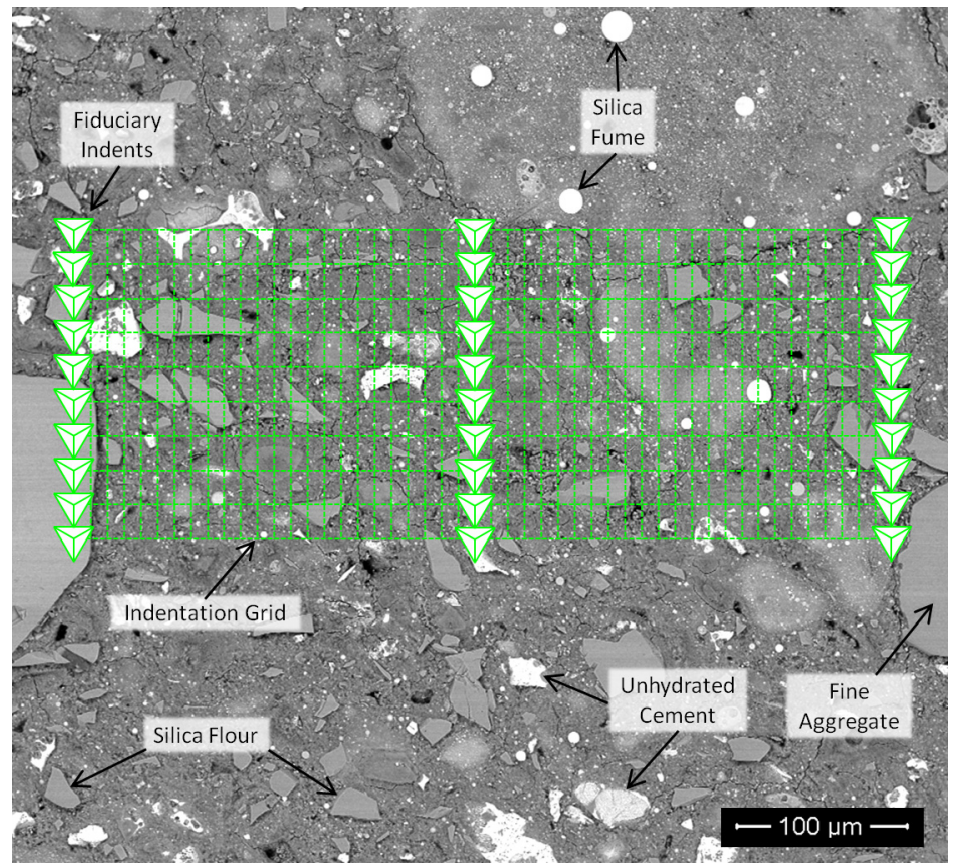

(a) SEM-BSE micrograph of typical RPC microstructure with measurement indentation grid and fiduciary indents superimposed.
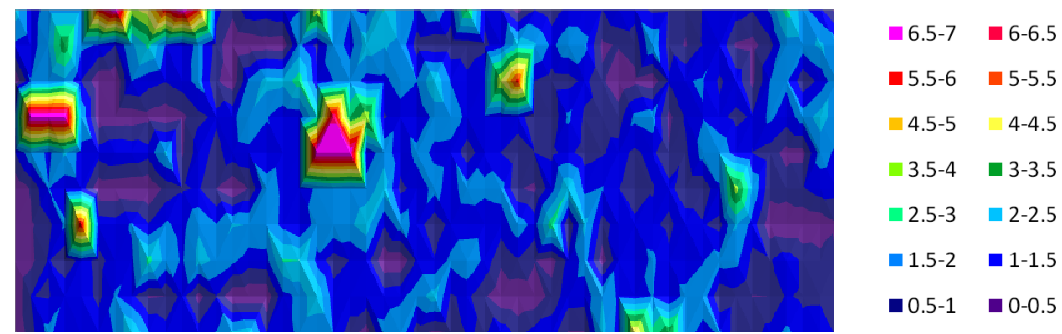

(b) Map of Ca:Si corresponding to indentation grid.
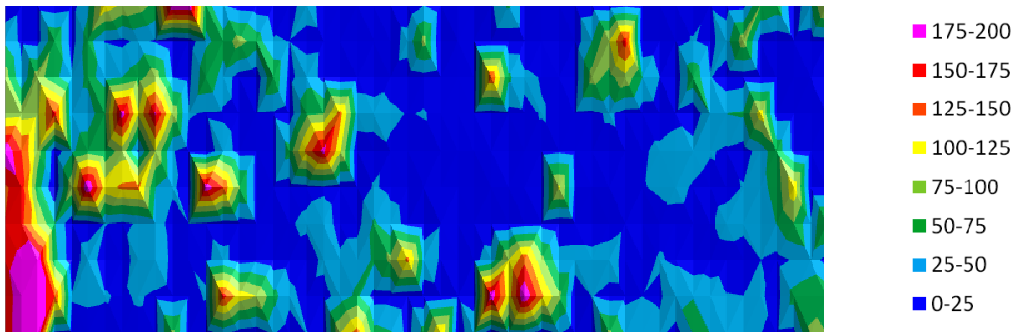

(c) Map of elastic modulus ( $\mathrm{GPa}$ ) corresponding to indentation grid.

Figure 3: Typical results from coupled nanoindentation and SEM/EDX studies performed on polished RPC specimens. 
Another feature of the nanoindentation measurements clearly shown in the elastic modulus contour map (see Figure 3 (c)) is the gradual transition in nanomechanical properties present at interfaces between two phases. This behavior is likely the result of a composite response of material present within the interaction region below each indent as discussed above.

With the aberrant results removed from the dataset, further analysis of "valid" nanoindentation results was performed. Figures 4 and 5 present histograms of the elastic modulus and hardness results of all "valid" indents performed on nonimpacted and impacted specimens in the present study. Results presented in Figures 4 encompass those of anhydrous cement, siliceous fine aggregates, silica flour, the HCP (primarily composed of C-S-H), and voids/porosity. Impacted specimens exhibited a mean elastic modulus of $47.9 \mathrm{GPa}$ compared with 76.7 GPa in non-impacted specimens. In particular, significant reductions in the proportion of indents with elastic moduli between $60 \mathrm{GPa}$ and $110 \mathrm{GPa}$ was observed in impacted specimens, a range common for silica flour and siliceous fine aggregates [10].

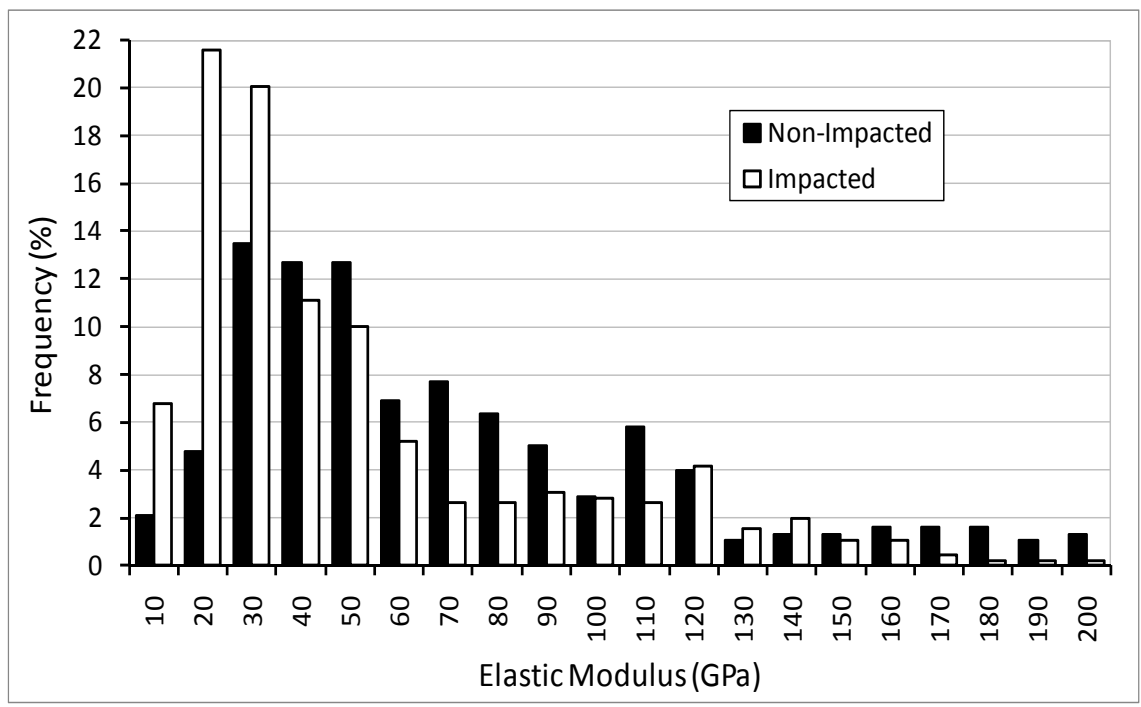

Figure 4: Histogram of elastic modulus results from nanoindentation experiments performed on non-impacted and impacted RPC specimens.

The effect of impact (Figure 5) was much more pronounced in hardness measurements, where a large shift in hardness from a mean of $6.3 \mathrm{GPa}$ to $2.7 \mathrm{GPa}$ was observed. In impacted specimens, there was a particularly high increase in the proportion of indents with hardness between 0.5 and $1.5 \mathrm{GPa}$ associated with a reduction in the proportion of indents with hardness above $4 \mathrm{GPa}$. 


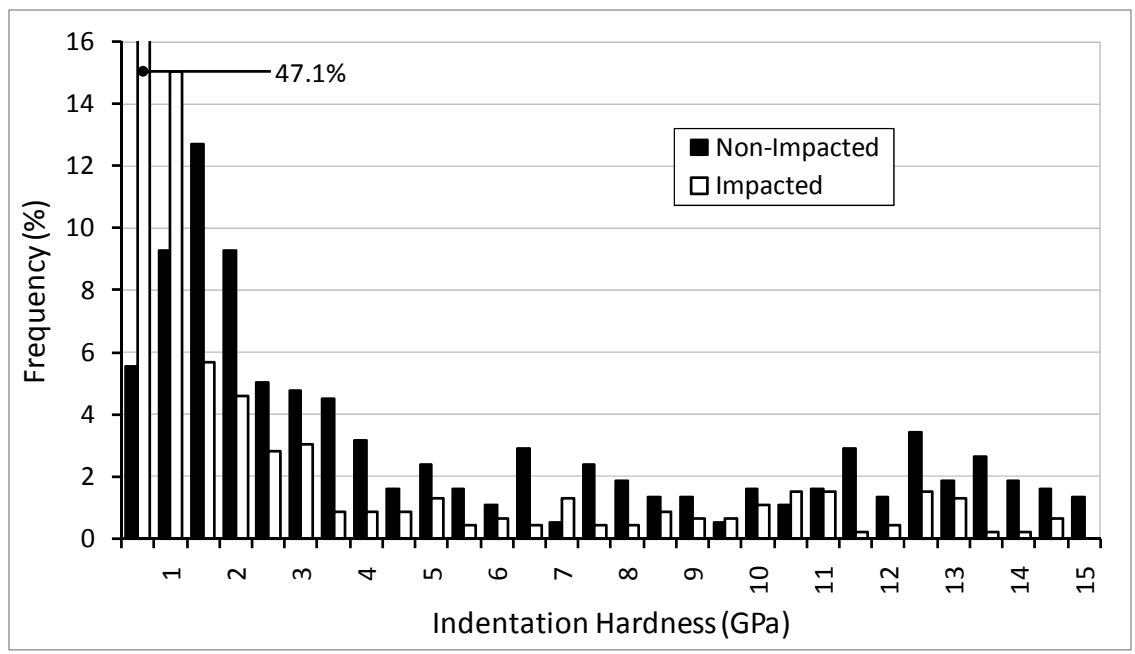

Figure 5: Histogram of indentation hardness results from nanoindentation experiments performed on non-impacted and impacted RPC specimens.

While these nanoindentation results suggest that impact loadings have a significant influence on both the elastic modulus and hardness of RPCs, it is difficult to determine by what possible mechanism degradation occurs. In order to further evaluate the possible mechanisms causing the observed degradation in nanomechanical properties, indents likely associated with homogenous C-S-H regions were extracted from the full dataset of non-impacted and impacted RPC specimens. Indents identified as C-S-H were selected based on a Ca:Si between 1 and 2 (consistent with C-S-H) with an elastic modulus between 10 and $50 \mathrm{GPa}$ (range typical for LD and HD C-S-H [10, 13]. Out of each dataset, approximately $10 \%$ of indents were deemed as homogenous C-S-H. Ca:Si ratios of the C-S-H sub-dataset were 1.44 and 1.42 for non-impacted and impacted specimens, respectively.

The histograms shown in Figures 6 and 7 depict the distribution in C-S-H elastic modulus and indentation hardness for non-impacted and impacted specimens. C-S-H present in non-impacted specimens exhibited a mean elastic modulus of $32.5 \mathrm{GPa}$ and mean indentation hardness of $1.56 \mathrm{GPa}$. C-S-H present in impacted specimens exhibited a mean elastic modulus of $27.4 \mathrm{GPa}$ and mean indentation hardness of $0.66 \mathrm{GPa}$, with a significant increase in hardness between 0.25 and $0.75 \mathrm{GPa}$.

These reductions in elastic modulus and, in particular, hardness in impacted samples suggests that the $\mathrm{C}-\mathrm{S}-\mathrm{H}$, in addition to the overall microstructure of RPCs, is degraded under high strain-rate impact loadings. The dehydration/vaporization mechanism proposed by DeJong and Ulm [8] was shown as a possible deterioration mechanism resulting in decreased C-S-H packing factors and in turn reduced elastic modulus and hardness as measured by nanoindentation. However, unless additional secondary chemical bonding was to 
occur between C-S layers (similar to irreversible creep mechanisms [16] during the vaporization event, it is likely that rehydration of the C-S-H would occur slowly. Furthermore, if such bonding were to occur; densifying the C-S-H and limiting rehydration, the elastic modulus and hardness would likely increase rather than decrease. Based on the results presented in Figures 4 and 5, it is clear that a majority of degradation in the full RPC indentation dataset is associated with reductions in elastic modulus and hardness associated with inert particles and unhydrated cement. Therefore, it is likely that a majority of degradation in RPCs following impact loading results from microfracturing and/or debonding.

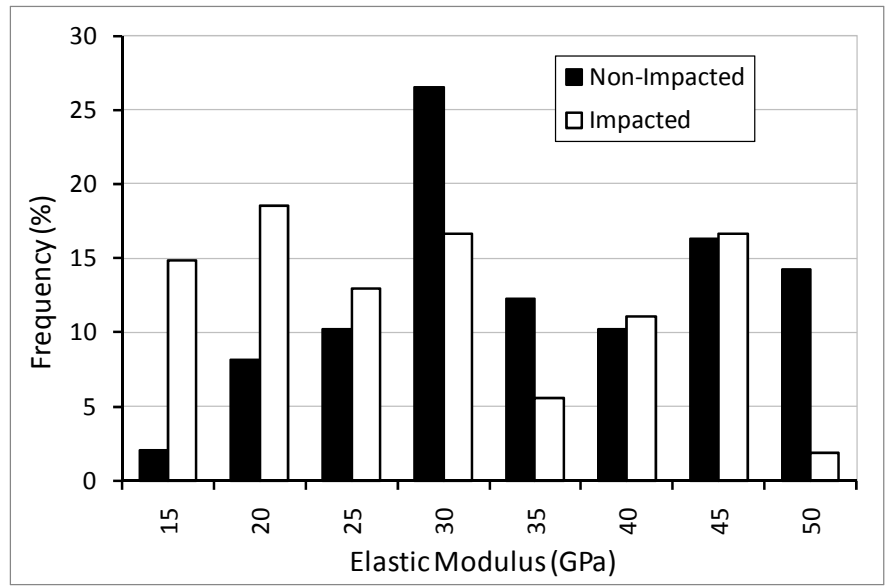

Figure 6: C-S-H elastic modulus of impacted and non-impacted specimens.

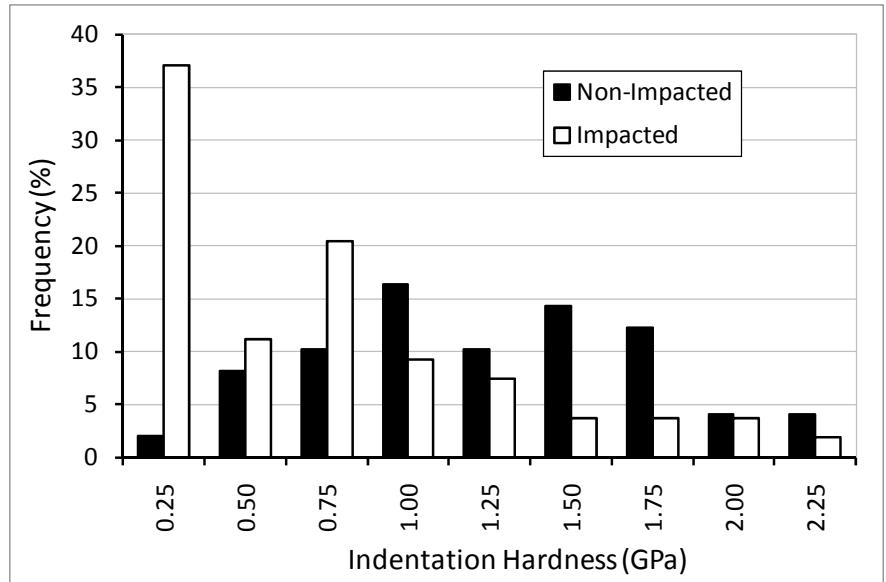

Figure 7: C-S-H indentation hardness of impacted and non-impacted specimens. 


\section{Conclusions}

A novel statistical nanoindentation technique was developed that spatially correlated the location of indents performed with an image and chemical analyses using EDX. Using this method, the influence of high strain-rate impact loadings on the nanomechanical properties of RPCs was determined. Significant degradation in both the elastic modulus and hardness occurred due to impact loadings of RPCs. Closer examination of C-S-H in particular also showed degradation. However, a link between chemical changes in the C-S-H and deterioration in nanomechanical properties could not be made. Based on analysis of all indents performed on RPC specimens, it is likely that a much of the degradation in elastic modulus and hardness observed stems from microfracturing and debonding, which occurs due to the impact.

Future work involves additional experimental studies and quantitative data analysis to further evaluate the possible mechanisms of degradation present in RPCs.

\section{Acknowledgement}

Permission to publish this article was granted by Director, Geotechnical \& Structures Laboratory.

\section{References}

[1] Millard, S.G., et al., Dynamic enhancement of blast-resistant ultra high performance fibre-reinforced concrete under flexural and shear loading. International Journal of Impact Engineering, 2010. 37: p. 405-413.

[2] Rushing, T.S., et al., Independent effects of matrix strength and fiber reinforcement on concrete's ballistic resistance. SAVIAC.

[3] Constantinides, G. and F.-J. Ulm, The nanogranular nature of C-S-H. Journal of the Mechanics and Physics of Solids, 2007. 55: p. 65-90.

[4] Grady, D., Shock equation of state properties of concrete, in Proc. of Structures under Shock and Impact IV, N. Jones et al., Editor. 1996, Computational Mechanics Publications: Southampton, UK. p. 405-414.

[5] Velez, K., et al., Determination by nanoindentation of elastic modulus and hardness of pure constituents of Portland cement clinker. Cement and Concrete Research, 2001. 31: p. 555-561.

[6] Hughes, J.J. and P. Trtik, Micro-mechanical properties of cement paste measured by depth-sensing nanoindentation: a preliminary correlation of physical properties with phase type. Materials Characterization, 2004. 53: p. 223-231.

[7] Ulm, F.-J., et al., Statistical indentation techniques for hydrated nanocomposites: concrete, bone, and shale. Journal of the American Ceramic Society, 2007. 90(9): p. 2677-2692. 
[8] DeJong, M.J. and F.-J. Ulm, The nanogranular behavior of $\mathrm{C}-\mathrm{S}-\mathrm{H}$ at elevated temperatures (up to $700{ }^{\circ} \mathrm{C}$ ). Cement and Concrete Research, 2007. 37(1): p. 1-12.

[9] Constantinides, G. and F.-J. Ulm, The effect of two types of C-S-H on the elasticity of cement-based materials: Results from nanoindentation and micromechanical modeling. Cement and Concrete Research, 2004. 34: p. 67-80.

[10] Sorelli, L., et al., The nano-mechanical signature of ultra high performance concrete by statistical nanoindentation techniques. Cement and Concrete Research, 2008. 38(12): p. 1447-1456.

[11] MIL-STD-662F, V50 ballistic test for armor. 1997.

[12] liver, W.C. and G.M. Pharr, Measurement of hardness and elastic modulus by instrumented indentation: Advances in understanding and refinements to methodology. Journal of Materials Research, 2004. 19(1): p. 3-20.

[13] Chen, J.J., et al., A coupled nanoindentation/SEM-EDS study on low water/cement ratio Portland cement paste: evidence for $\mathrm{C}-\mathrm{S}-\mathrm{H} / \mathrm{Ca}(\mathrm{OH}) 2$ nanocomposites. Journal of the American Ceramic Society, 2010. 93(5): p. 1484-1493.

[14] Davydov, D., M. Jirasek, and L. Kopecky, Critical aspects of nanoindentation technique in application to hardened cement paste. Cement and Concrete Research, 2010.

[15] Trtik, P., B. Munch, and P. Lura, A critical examination of statistical nanoindentation on model materials and hardened cement pastes based on virtual experiments. Cement \& Concrete Composites, 2009. 31: p. 705-714.

[16] Mehta, P.K. and P.J.M. Monteiro, Concrete: Microstructure, Properties, and Materials. 2006: McGraw-Hill Companies Ltd. 